\title{
An Out-of-Sample Extension for Wireless Multipoint Channel Charting
}

\section{Conference Paper}

\section{Author(s):}

Ponnada, Tushara; Al-Tous, Hanan; Tirkkonen, Olav; Studer, Christoph (D)

\section{Publication date:}

2019

\section{Permanent link:}

https://doi.org/10.3929/ethz-b-000461386

\section{Rights / license:}

In Copyright - Non-Commercial Use Permitted

\section{Originally published in:}

Lecture Notes of the Institute for Computer Sciences, Social Informatics and Telecommunications Engineering 291, https:// doi.org/10.1007/978-3-030-25748-4_16 


\title{
An Out-of-Sample Extension for Wireless Multipoint Channel Charting
}

\author{
Tushara Ponnada ${ }^{1}$, Hanan Al-Tous ${ }^{1}$, Olav Tirkkonen ${ }^{1}$, and Christoph Studer ${ }^{2}$ \\ ${ }^{1}$ Department of Communications and Networking, Aalto University, Espoo, Finland \\ ${ }^{2}$ School of Electrical and Computer Engineering, Cornell University, Ithaca, NY, \\ USA \\ \{tushara.ponnada, hanan.al-tous, olav.tirkkonen\}@aalto.fi, \\ studer@cornell.edu
}

\begin{abstract}
Channel-charting (CC) is a machine learning technique for learning a multi-cell radio map, which can be used for cognitive radioresource-management (RRM) problems. Each base-station (BS) extracts features from the channel-state-information samples (CSI) from transmissions of user-equipment (UE) at different unknown locations. The multi-path channel components are estimated and used to construct a dissimilarity matrix between CSI samples at each BS. A fusion center combines the dissimilarity matrices of all base-stations, performs dimensional reduction based on manifold learning, constructing a MultipointCC (MPCC). The MPCC is a two dimension map, where the spatial difference between any pair of UEs closely approximates the distance between the clustered features. MPCC provides a mapping for any given trained UE location. To use MPCC for cognitive RRM tasks, CSI measurements for new UEs would be acquired, and these UEs would be placed on the radio map. Repeating the MPCC procedure for out-ofsample CSI measurements is computationally expensive. For this, extensions of MPCC to out-of-sample UE CSIs are investigated in this paper, when Laplacian-Eigenmaps (LE) is used for dimensional reduction. Simulation results are used to show the merits of the proposed approach.
\end{abstract}

Keywords: Massive MIMO Channel Charting - Laplacian Eigenmaps. Out-of-Sample Mapping.

\section{Introduction}

Massive-multiple-input-multiple-output (mMIMO) technology is a promising technology for fifth-generation $(5 \mathrm{G})$ cellular communications, with the potential to provide high spectral and power efficiency. In a mMIMO cell, each base-station (BS) has a large number of antennas, which can provide a simultaneous use of the resource (e.g., frequency and/or time slots) for multiple user equipment (UEs) in the cell $[3,4,13]$. Furthermore, the high spatial resolution exploited by the large-scale antenna arrays used at the mMIMO BSs can be used for many applications, such as UE positioning and environment mapping $[7,8,11]$. 
To efficiently mange a mMIMO network, and to perform cognitive networking tasks, the network states which include the spatial distribution and trajectories of the UEs, neighborhood relationships among the UEs, and handover boundaries among neighboring cells need to be estimated. A novel framework called channel charting (CC) based on the massive amounts of channel-state-information (CSI) available at the base-stations is proposed for a single cell MIMO system in [12]. $\mathrm{CC}$ is based on using unsupervised machine learning techniques to create a radio map of the cell served by the BS, which preserves the neighborhood relations of UEs, using features that characterize the large scale fading effects of the channel. The obtained CC can be used for local radio-resource-management (RRM) in the cell. However, cell edge UEs may not be accurately located in the chart due to their low signal-to-noise-ratio (SNR) at the cell edge.

In [5], a multi-point CC (MPCC) framework is proposed to support advanced multi-cell RRM and to accurately map cell edge UEs. For improved charting performance, features are extracted and clustered based on advanced signal processing and machine learning techniques. Each BS generates it own dissimilarity matrix between the users it can decode, then the dissimilarity matrices are fused at a fusion center and then used to construct the MPCC. The trustworthiness and continuity measures show that the proposed MPCC is capable to preserve the neighborhood structure between UEs in the network.

To use the MPCC framework for different RRM functionalities, it is important to generalize its capability, allowing to incorporate new data to an existing MPCC and/or to estimate the features related to a location in the chart. As the CSI of a UE can change rapidly in a small distance, it is important to accurately estimate the location of an out-of-sample UE location. In this paper, the extension of MPCC to out-of-sample data points based on Laplacian Eigenmaps (LE) is considered.

The remainder of this paper is organized as follows. In Section 2 and Section3, the system model and the MPCC are introduced, respectively. In Section 4, the problem formulation is presented. Numerical results are presented and discussed in Section 5. Finally, conclusions are drawn in Section 6.

\section{System Model}

The system under consideration is schematically shown in Fig. 1. Each BS $b$ for $b=1, \cdots, B$ has $M$ antenna elements and each UE $k$ for $k=1, \cdots, K$ has a single antenna element. For a mMIMO system, the channel vector of UE $k$ using a uniform-linear-array (ULA) at BS $b$ for a coherence bandwidth can be modeled as $[5]$ :

$$
\boldsymbol{h}_{b}^{(k)}=\sum_{l=1}^{L_{k}} \beta_{b}^{(k)}(l) \boldsymbol{a}_{b}\left(\phi_{b}^{(k)}(l)\right),
$$

where $L_{k}$ is the number of multi-path components for the wireless channel between $\mathrm{UE} k$ and $\mathrm{BS} b, \phi_{b}^{(k)}(l)$ is the direction of arrival of the $l$ th path and $\beta_{b}^{(k)}(l)$ 
is the gain of the $l$ th path, and $\boldsymbol{a}_{b}(\cdot)$ is BS $b$ steering vector. For ULA, $\boldsymbol{a}(\phi)$ can be modeled as:

$$
\boldsymbol{a}(\phi)=\left[1, \mathrm{e}^{2 \frac{2 \pi}{\lambda} s \sin (\phi)}, \cdots, \mathrm{e}^{2 \frac{2 \pi}{\lambda}(M-1) s \sin (\phi)}\right]^{T},
$$

where $\lambda$ is the carrier wave-length and $s$ is antenna spacing. The covariance $\boldsymbol{Y}_{b}^{(k)} \in \mathbb{C}^{M \times M}$ of the CSI $\boldsymbol{h}_{b}^{(k)}$ used to extract the features becomes

$$
\boldsymbol{Y}_{b}^{(k)}=\mathbb{E}\left[\boldsymbol{h}_{b}^{(k)} \boldsymbol{h}_{b}^{(k) H}\right]=\boldsymbol{A}_{b}^{(k)} \boldsymbol{S}_{b}^{(k)} \boldsymbol{A}_{b}^{(k) H},
$$

where $\mathbb{E}$ is the expectation operator, $\boldsymbol{A}_{b}^{(k)}=\left[\boldsymbol{a}\left(\phi_{b}^{(k)}(1)\right), \cdots, \boldsymbol{a}\left(\phi_{b}^{(k)}\left(L_{k}\right)\right)\right]$ is a matrix of array steering vectors, and $\boldsymbol{S}_{b}^{(k)}=\operatorname{diag}\left(\mathbb{E}\left[\left|\beta_{b}^{(k)}(1)\right|^{2}\right], \cdots\right.$, $\left.\mathbb{E}\left[\left|\beta_{b}^{(k)}\left(L_{k}\right)\right|^{2}\right]\right)$ is a diagonal matrix of multi-path power components. Channel charting is based on the assumption that there is a continuous mapping from the spatial location $\boldsymbol{p}^{(k)}$ of UE $k$ to the covariance $\operatorname{CSI} \boldsymbol{Y}_{b}^{(k)}$ given as $[5,12]$ :

$$
\mathcal{H}_{b}: \mathbb{R}^{d} \rightarrow \mathbb{C}^{M \times M} ; \mathcal{H}_{b}\left(\boldsymbol{p}^{(k)}\right)=\boldsymbol{Y}_{b}^{(k)},
$$

where $d$ is the spatial dimension.

\section{$3 \quad \mathrm{MPCC}$}

Multi-point channel charting extends CC to multiple BSs. A block diagram representing MPCC is shown in Fig. 2. Using the estimated covariance CSI $\left\{\left\{\boldsymbol{Y}_{b}^{(k)}\right\}_{k=1}^{K}\right\}_{b=1}^{B}$ collected at $B$ BSs form $K$ unknown UE spatial locations $\boldsymbol{P}=\left\{\boldsymbol{p}_{k}\right\}_{k=1}^{K}$, the MPCC finds a low dimension channel chart $\boldsymbol{Z}=\left\{\boldsymbol{z}_{k}\right\}_{k=1}^{K}$, such that:

$$
\left\|\boldsymbol{z}_{k}-\boldsymbol{z}_{m}\right\| \approx \alpha\left\|\boldsymbol{p}_{k}-\boldsymbol{p}_{m}\right\|, \text { for } k, m \in\{1, \cdots, K\},
$$

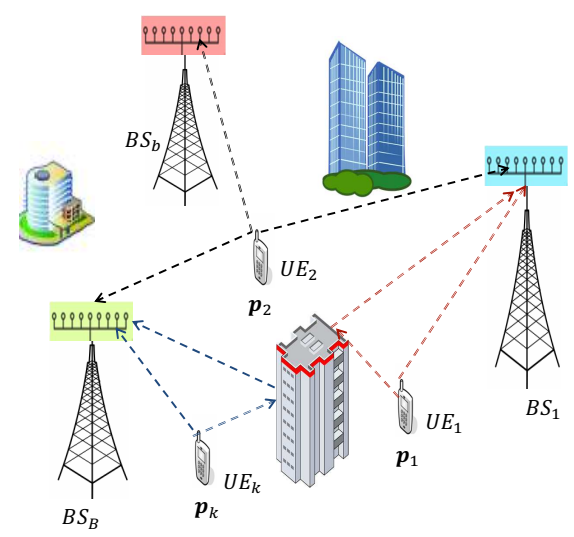

Fig. 1: Multipoint mMIMO system. 


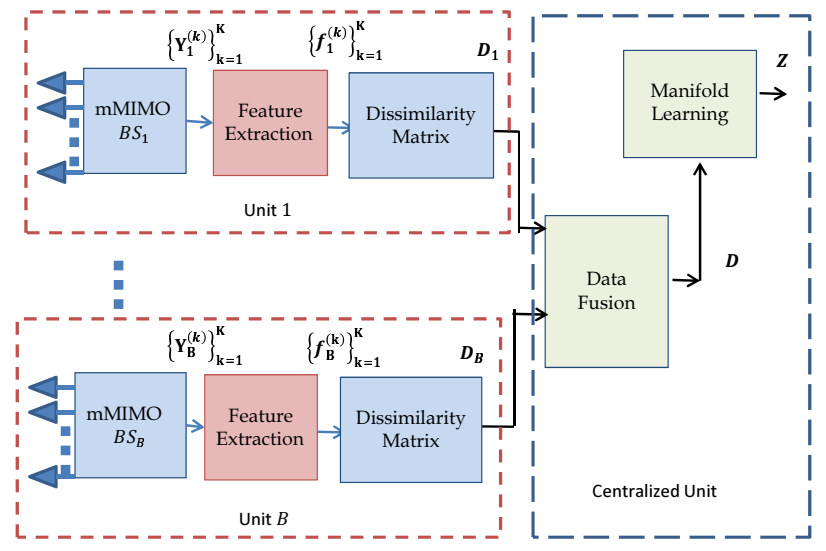

Fig. 2: MPCC block diagram.

where $\alpha$ is a scaling factor. Note that neither the UEs spatial locations $\boldsymbol{P}$ nor the locations of the BSs are known; the MPCC is computed solely based on the covariance CSI $\left\{\left\{\boldsymbol{Y}_{b}^{(k)}\right\}_{k=1}^{K}\right\}_{b=1}^{B}$.

\subsection{Feature Extraction and Dissimilarity Matrix}

The feature vector $\boldsymbol{f}_{b}^{(k)}$ for UE $k$ at BS $b$ is selected based on the multi-path components as [5]:

$$
\boldsymbol{f}_{b}^{(k)}=\left[\lambda_{b}^{(k)}(1), \cdots, \lambda_{b}^{(k)}\left(L_{k}\right), \phi_{b}^{(k)}(1), \cdots, \phi_{b}^{(k)}\left(L_{k}\right)\right],
$$

where $\lambda_{b}^{(k)}(l)=\mathbb{E}\left[\left|\beta_{b}^{(k)}(l)\right|^{2}\right]$. The multi-path components (power and phase) $\left\{\lambda_{b}^{(k)}(l)\right\}_{l=1}^{L_{k}}$ and $\left\{\phi_{b}^{(k)}(l)\right\}_{l=1}^{L_{k}}$ of UE $k$ at BS $b$ are estimated from the covariance matrix $\boldsymbol{Y}_{b}^{(k)}$ using the multiple-signal-classification (MUSIC) algorithm [10]. The dissimilarity between two UEs $(k, m)$ is based on identifying multi-path components in their feature vectors that are similar. For this, the components of feature vectors are transformed to Cartesian coordinates as [5]:

$$
\mathcal{F}\left\{\boldsymbol{f}_{b}^{(k)}\right\}=\left[\boldsymbol{x}_{b}^{(k)}(1), \cdots, \boldsymbol{x}_{b}^{(k)}\left(L_{k}\right)\right],
$$

where $\boldsymbol{x}_{b}^{(k)}(l)=\left[\lambda_{b}^{(k)}(l) \cos \left(\phi_{b}^{(k)}(l)\right), \lambda_{b}^{(k)}(l) \sin \left(\phi_{b}^{(k)}(l)\right)\right]^{T}$.

A 2D non-linear transformation $\mathcal{N}_{b}^{\nu}: \mathbb{R}^{2} \rightarrow \mathbb{R}^{2}$ with a set of parameters $\boldsymbol{\nu}$ is hand-crafted based on the statistical and geometrical characteristics of the multi-path components of all $K$ UEs that a BS is seeing. This function is applied to the geometrical representations of the multi-path components $\boldsymbol{x}_{b}^{(k)}(l)$. The 
transformation $\mathcal{N}_{b}^{\nu}$ is used in order to make clusters of multipath components separable.

To cluster multipath components to clusters deemed to be similar, the densitybased-spatial-clustering-of-applications-with-noise (DBSCAN) algorithm [6] is used to label the multi-path components after applying the transformation $\mathcal{N}_{b}^{\nu}$. This results in a label $\mathcal{L}\left(\mathcal{N}_{b}^{\nu}\left(\boldsymbol{x}_{b}^{(k)}(i)\right)\right) \in\left\{C_{1}, \cdots, C_{N}\right\}$ for each multi-path component, where $C_{n}$ is the label of the $n$th cluster. The dissimilarity coefficient between a pair of UEs $(k, m)$ then is computed taking into consideration multi-path components of the UEs that are in the same cluster. Dissimilarity is computed as:

$$
\begin{aligned}
& d_{b}\left(\boldsymbol{f}_{b}^{(k)}, \boldsymbol{f}_{b}^{(m)}\right) \\
& \quad= \begin{cases}\left\|\boldsymbol{x}_{b}^{(k)}\left(i^{*}\right)-\boldsymbol{x}_{b}^{(m)}\left(j^{*}\right)\right\|_{2} & \text { if } \mathcal{L}\left(\mathcal{N}_{b}^{\nu}\left(\boldsymbol{x}_{b}^{(k)}\left(i^{*}\right)\right)\right)=\mathcal{L}\left(\mathcal{N}_{b}^{\nu}\left(\boldsymbol{x}_{b}^{(m)}\left(j^{*}\right)\right)\right), \\
\left\|\boldsymbol{x}_{b}^{(k)}(1)-\boldsymbol{x}_{b}^{(m)}(1)\right\|_{2}, & \text { otherwise, }\end{cases}
\end{aligned}
$$

where $\left[i^{*}, j^{*}\right]=\underset{i, j}{\operatorname{argmin}}\left(\lambda_{b}^{(k)}(i), \lambda_{b}^{(m)}(j)\right)$. The dissimilarity matrix $\boldsymbol{D}_{b} \in \mathbb{R}^{K \times K}$ then has the elements $D_{b}(k, m)=d_{b}\left(\boldsymbol{f}_{b}^{(k)}, \boldsymbol{f}_{b}^{(m)}\right)$ for $k, m=1, \cdots, K$.

The benefits of having multiple spatially distributed BSs can be utilized by merging the BS-specific dissimilarity matrices $\left\{\boldsymbol{D}_{b}\right\}_{b=1}^{B}$ into a global dissimilarity matrix $\boldsymbol{D}$, where the $(k, m)$ th element $D(k, m)$ can be computed as:

$$
D(k, m)=\frac{1}{\sum_{b=1}^{B} \omega_{b}(k, m)} \sum_{b=1}^{B} \omega_{b}(k, m) D_{b}(k, m),
$$

where $\omega_{b}(k, m)$ is a weighting factor computed as $\omega_{b}(k, m)=\min \left(\gamma_{b}^{(k)}, \gamma_{b}^{(m)}\right)^{2}$ and $\gamma_{b}^{(k)}$ is the SNR of the wireless link between UE $k$ and BS $b$.

\section{Out-of-sample Extension}

For a given dissimilarity matrix, different dimension reduction techniques (i.e., linear, non-linear, convex and non-convex optimization approaches) have been proposed in the literature. The performance of a given technique is problem dependent, as discussed in [9]. The single cell CC problem has been solved using the principle-component-analysis (PCA), Sammon's-mapping (SM) and Autoencoder reduction techniques in [12], whereas the MPCC is solved using SM, Laplacian Eigenmaps (LE) and $t$-distributed-stochastic-neighbor-embedding ( $t$ $\mathrm{SNE}$ ) in [5]. In this paper, LE is considered, and extended for the out-of-sample MPCC problem.

LE is a computationally efficient non-linear dimensionality reduction algorithm based on the graph Laplacian, that preserves neighborhood properties and clustering connections [1]. LE constructs a graph from neighborhood information 


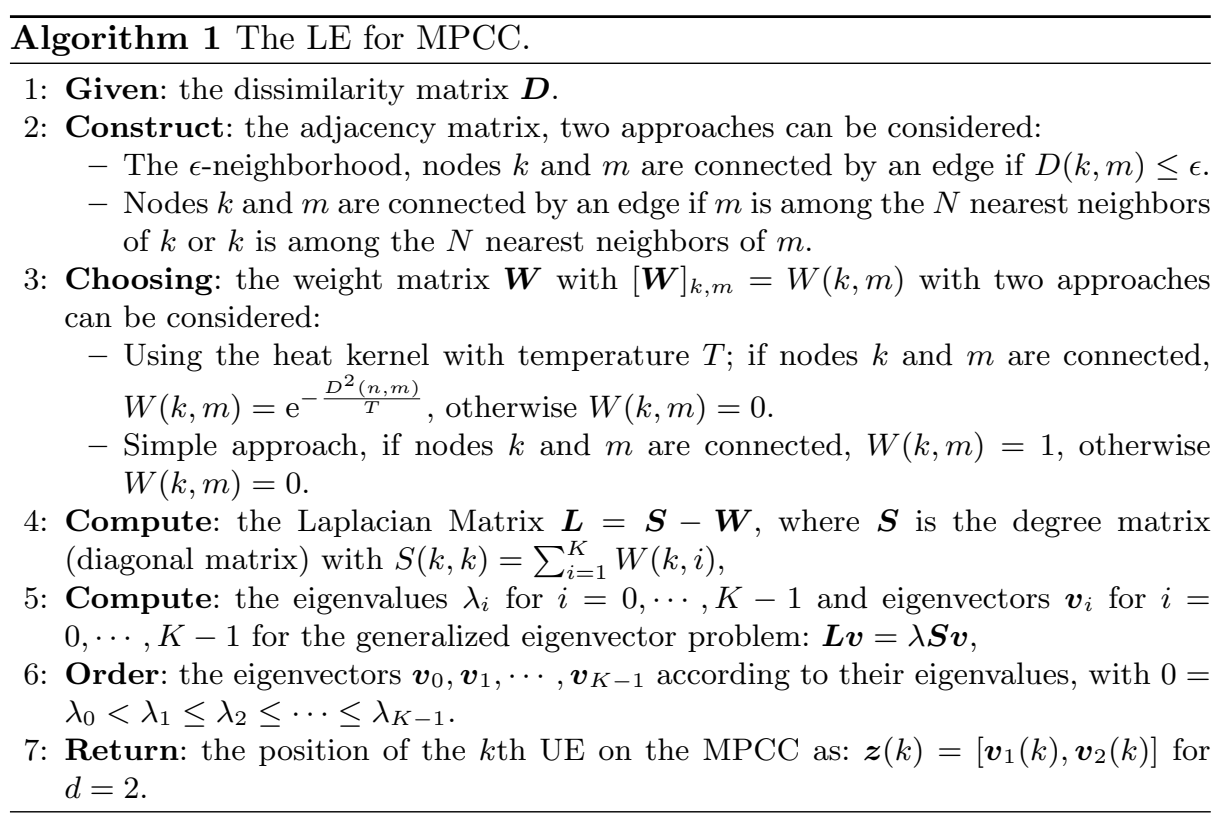

of the dissimilarity matrix. The LE problem can be formulated as [1]:

$$
\begin{aligned}
& \min _{\boldsymbol{X}} \operatorname{trace}\left(\boldsymbol{X}^{T} \boldsymbol{L} \boldsymbol{X}\right), \\
& \text { s.t. } \boldsymbol{X}^{T} \boldsymbol{S} \boldsymbol{X}=\boldsymbol{I}_{K},
\end{aligned}
$$

where trace is the trace function, $\boldsymbol{I}_{K}$ is the identity matrix of order $K, \boldsymbol{X}=$ $\left[\boldsymbol{x}(1)^{T}, \cdots, \boldsymbol{x}(K)^{T}\right]^{T}$ represents the optimization variables in a matrix form, $\boldsymbol{L}$ is the Laplacian matrix and $\boldsymbol{S}$ is the degree matrix as detailed below. The solution of (10) can be obtained in a closed form as the solution of a generalized eigenvector problem [1].

The MPCC is obtained by computing the eigenvectors of the LE as described in Algorithm 1. Since the MPCC is constructed by processing the data of all UEs from all BSs, it is computationally expensive to repeat the MPCC process if an out-of-sample data item is available, and needs to be inserted into the chart. If the original MPCC is based on a sufficient number of samples, it is expected that the out-of-sample data will not change the MPCC positions.

Here, we address out-of-sample extension of MPCC in this sense, aiming to estimate the location of the new sample on the MPCC, to be used for RRM functions, such as hand-over prediction. It is worth mentioning that an out-ofsample data item needs to be processed using the same non-linear transformation $\mathcal{N}_{b}^{\nu}$ at each BS $b$ for $b=1, \cdots, B$ and then, the cluster labeling based on the original data has to be applied for each multi-path component.

In [2], a generalized framework for out-of-sample extension is proposed for several algorithms, providing that these algorithms can be seen as learning eigenfunctions of a data dependent kernel. The out-of-sample mapping can be formu- 


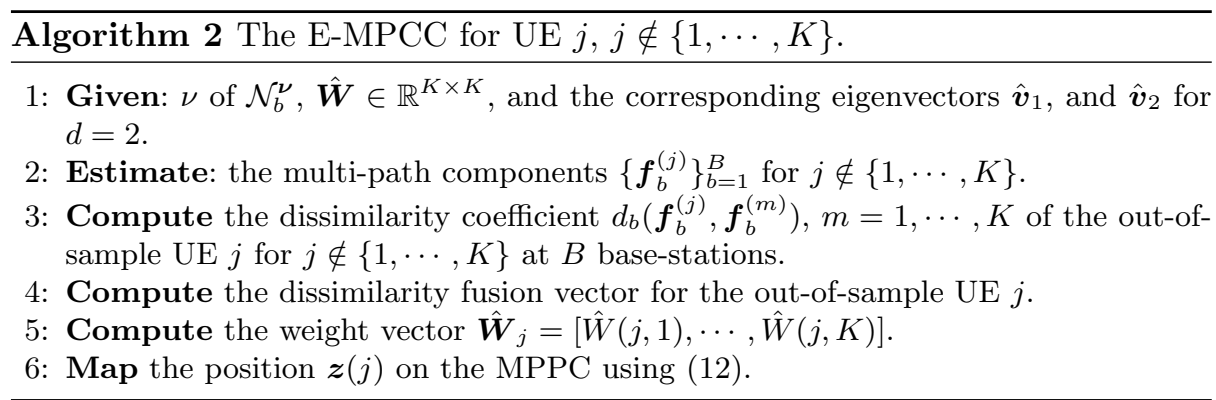

lated as an optimization problem, where the objective is to find a normalized kernel function that minimizes the mean squared-error. The normalized kernel vector is used as a weight vector to find the out-of-sample mapping. Using this on MPCC is called E-MPCC. For LE, the normalized kernel functions (weights) are computed as [2]:

$$
\hat{W}(k, i)=\frac{1}{K} \frac{W(k, i)}{\sqrt{\mathbb{E}_{x}[W(k, x)] \mathbb{E}_{y}[W(i, y)]}}, k, i \in\{1, \cdots, K\},
$$

where the expectation is taking with respect to the original data set. The EMPCC position of an out-of-sample data $\boldsymbol{z}(j)$ for $j \notin\{1, \cdots, K\}$ can be computed as:

$$
\boldsymbol{z}(j)=\left[\sum_{k=1}^{K} \hat{W}(j, k) \hat{\boldsymbol{v}}_{1}(k), \sum_{k=1}^{K} \hat{W}(j, k) \hat{\boldsymbol{v}}_{2}(k)\right],
$$

where the weight $\hat{W}(j, i)$ for $j \notin\{1, \cdots, K\}$ is computed based on the dissimilarity of the location with respect to the points in the original set, and the eigenvectors $\hat{\boldsymbol{v}}_{1}$ and $\hat{\boldsymbol{v}}_{2}$ are computed based on the normalized weighting matrix $\hat{W}$.

\section{Simulation Results}

An urban outdoor multi-cell mmWave scenario is considered as discussed in [5]. The system parameters are shown in Table 1. A ray tracing channel model is used to generate multi-path channels. We generate $K$ UE locations on the streets of a Manhattan grid. The CSI of the UEs are estimated at multiple BSs. The number of nearest neighbors $N$ are selected as $5 \%$ of UEs. The number of new samples $J$ for which out-of-sample extension is applied is $10 \%$ of the total number of UEs.

Two scenarios are considered. In Scenario I, the MPCC is generated based on the channel features of $K \mathrm{UE}$ locations, and then $J \mathrm{UE}$ locations are removed randomly. The proposed E-MPCC is used for mapping the $J$ locations to the chart. In scenario II, $J$ UE locations are selected randomly and the MPCC is generated based on the channel features of $K-J$ UE locations. The proposed E-MPCC is used for mapping the $J$ locations to the chart. 
An example instance for MPCC/-EMPCC of Scenarios I\&II for different settings is shown in Fig. 3. Clearly, the depicted chart shows that the $J$ outof-sample locations are accurately mapped by E-MPCC. For settings 1 (Set. 1), the parameters are $K=500, J=100$ and $B=4$, and for settings 2 (Set. 2), the parameters are $K=5000, J=500$ and $B=10$. The performance of the E-MPCC is evaluated using the continuity $(\mathrm{CT})$ and trustworthiness (TW) measures as shown in Table 2. For a discussion on these measures, see [12]. The $\mathrm{CT}$ and TW measures of the E-MPCC are comparable of the MPCC. The CT and TW are computed by considering 20 nearest neighbors. For MPCC, all $K$ $\mathrm{UE}$ are used to generate the chart, whereas for E-MPCC, the chart is constructed by $K-J$ UEs and the E-MPCC is used to position the reaming $J$ UEs.

The probability distribution of the relative-error $\epsilon_{r}$ of out-of-sample locations of E-MPCC is shown in Fig. 4. The probability that $\epsilon_{r}$ is less than $7 \%$ is $90 \%$. The small relative error is a promising indicator that E-MPCC can be used for different RRM functionalities.

Table 1: Simulation parameters [5].

\begin{tabular}{llll}
\hline Parameter & Value & Parameter & Value \\
\hline Carrier frequency & $28 \mathrm{GHz}$ & Bandwidth & $256 \mathrm{MHz}$ \\
UE Tx power & $23 \mathrm{dBm}$ & BS noise power & $-86 \mathrm{dBm}$ \\
OFDM subcarriers & 256 & & \\
\hline
\end{tabular}

Table 2: CT and TW performance measures.

\begin{tabular}{lllll}
\hline & \multicolumn{2}{c}{ MPCC } & \multicolumn{2}{c}{ E-MPCC } \\
\cline { 2 - 5 } & CT & TW & CT & TW \\
\hline Settings 1 & 0.725 & 0.682 & 0.7203 & 0.677 \\
Settings 2 & 0.755 & 0.701 & 0.758 & 0.701 \\
\hline
\end{tabular}

\section{Conclusion}

In this paper, Multipoint Channel Charting based on Laplacian Eigenmap manifold reduction was extended to out-of-sample UE locations. First, a MPCC was constructed using an original data set of UE CSIs. The multi-path components of the new CSI sample were estimated at each BS and then processed using the 


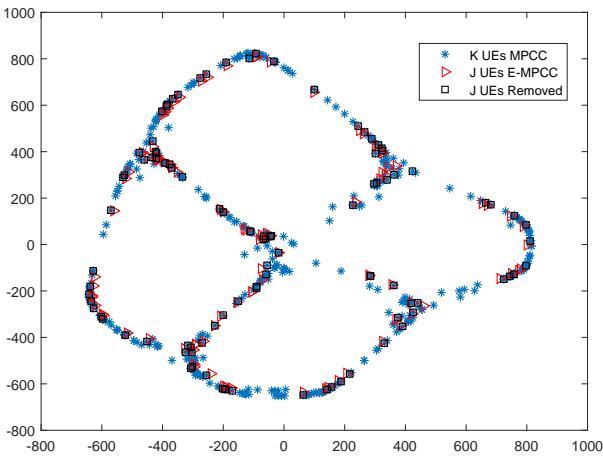

(a)

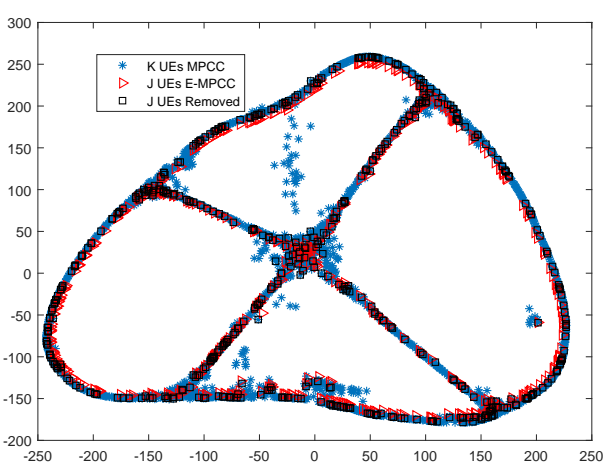

(c)

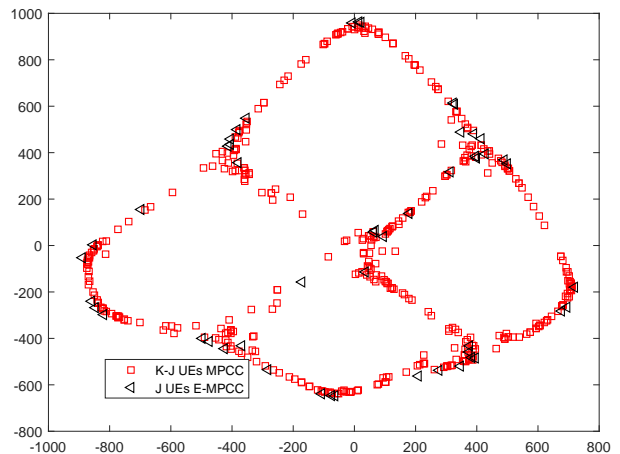

(b)

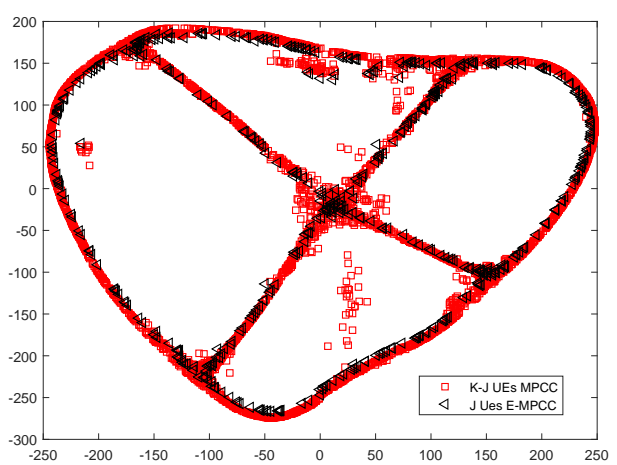

(d)

Fig. 3: E-MPCC and MPCC for: (a) Scenario I and Set. 1; (b) Scenario II and Set. 1; (c) Scenario I and Set. 2 (d) Scenario II and Set. 2.

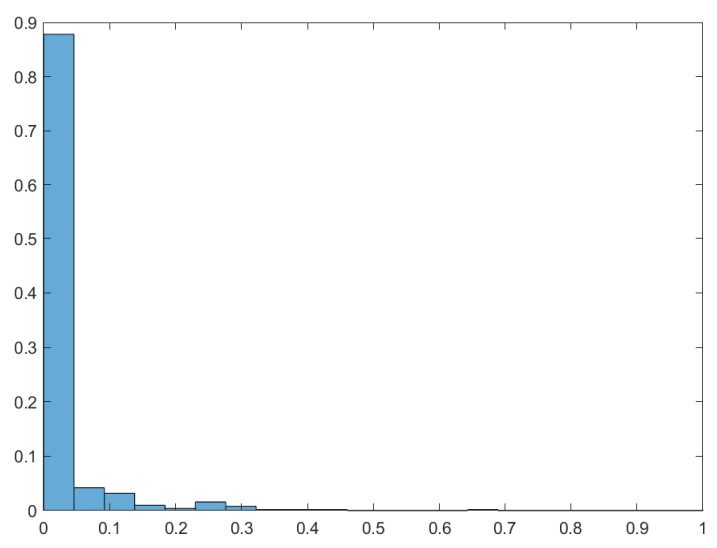

Fig. 4: The probability of relative-error $\epsilon_{r}$. 
same non-linear transformation as the original set. The dissimilarity vector of the out-of-sample UE is used to generate the weighting vector for out-of-sample mapping. The resulting E-MPCC algorithm is then used to map out-of-sample UEs to the MPCC map. The trustworthiness and continuity performance measures were used to evaluate the E-MPCC, and it was found that out-of-sample extension works in a reliable manner. The method has wide applicability in cognitive radio resource management, where predictions of UE connectivity parameters would be used.

In future work, the out-of-sample extension of MPCC for different dimension reduction techniques are going to be addressed. Machine learning techniques based on neural networks can be used to parametrize the MPCC and then used for out-of-sample mapping. The efficiency and accuracy of out-of-sample extensions need to be evaluated for different RRM functions such as handover and identifying cell boundaries.

\section{References}

1. Belkin, M., Niyogi, P.: Laplacian eigenmaps for dimensionality reduction and data representation. Neural Computation 15(6), 1373-1396 (June 2003)

2. Bengio, Y., Paiement, J.F., Vincent, P., Delalleau, O., Roux, N.L., Ouimet, M.: Out-of-sample extensions for lle, isomap, mds, eigenmaps, and spectral clustering. In: In Advances in Neural Information Processing Systems. pp. 177-184. MIT Press (2004)

3. Bjornson, E., Larsson, E.G., Marzetta, T.L.: Massive MIMO: ten myths and one critical question. IEEE Trans. Commun. 54(2), 114-123 (Feb 2016)

4. Busari, S.A., Huq, K.M.S., Mumtaz, S., Dai, L., Rodriguez, J.: Millimeter-wave massive MIMO communication for future wireless systems: A survey. IEEE Communications Surveys \& Tutorials 20(2), 836-869 (Second quarter 2018)

5. Deng, J., Medjkouh, S., Malm, N., Tirkkonen, O., Studer, C.: Multipoint channel charting for wireless networks. In: Proc. of 52nd Asilomar Conference on Signals, Systems, and Computers. pp. 286-290 (Oct 2018)

6. Ester, M., Kriegel, H.P., Sander, J., Xu, X.: A density-based algorithm for discovering clusters in large spatial databases with noise. pp. 226-231. AAAI Press (1996)

7. Garcia, N., Wymeersch, H., Larsson, E.G., Haimovich, A.M., Coulon, M.: Direct localization for massive MIMO. IEEE Trans. Signal Process. 65(10), 2475-2487 (May 2017)

8. Guidi, F., Guerra, A., Dardari, D., Clemente, A., D'Errico, R.: Environment mapping with millimeter-wave massive arrays: System design and performance. In: Proc. of IEEE Globecom Workshops (GC Wkshps). pp. 1-6 (Dec 2016)

9. van der Maaten, L., Postma, E.O., van den Herik, H.J.: Dimensionality reduction: A comparative review (2008)

10. Schmidt, R.: Multiple emitter location and signal parameter estimation. IEEE Trans. Antennas Propag. 34(3), 276-280 (March 1986)

11. Shahmansoori, A., Garcia, G.E., Destino, G., Seco-Granados, G., Wymeersch, H.: Position and orientation estimation through millimeter-wave MIMO in $5 \mathrm{G}$ systems. IEEE Trans. Wireless Commun. 17(3), 1822-1835 (March 2018) 
12. Studer, C., Medjkouh, S., Gnlta, E., Goldstein, T., Tirkkonen, O.: Channel charting: Locating users within the radio environment using channel state information. IEEE Access 6, 47682-47698 (2018)

13. Yang, S., Hanzo, L.: Fifty years of MIMO detection: The road to large-scale MIMOs. IEEE Communications Surveys \& Tutorials 17(4), 1941-1988 (Fourth quarter 2015) 